\title{
Prenatal exposure to acid suppressant medications and risk of allergen sensitization
}

\author{
Lacey B. Robinson ${ }^{1}$, Anna Arroyo ${ }^{2}$, Ruth J. Geller ${ }^{3}$, Ashley F. Sullivan ${ }^{3}$, and Carlos \\ Camargo $\mathrm{Jr}^{1}$ \\ ${ }^{1}$ Massachusetts General Hospital Division of Rheumatology Allergy and Immunology \\ ${ }^{2}$ Stanford University Division of Pulmonary and Critical Care Medicine \\ ${ }^{3}$ Massachusetts General Hospital
}

November 15, 2021

\begin{abstract}
In conclusion, in a cohort of children with a history of severe bronchiolitis those exposed to ASM prenatally are not at increased risk of developing food or environmental allergen sensitization by early childhood. The mechanism by which ASM exposure increases risk of childhood asthma remains unclear, but alterations in the gut microbiome merit consideration.
\end{abstract}

Title:Prenatal exposure to acid suppressant medications and risk of allergen sensitization

Lacey B. Robinson, MD, MPH${ }^{1}$; Anna Chen Arroyo, MD, $\mathrm{MPH}^{2}$; Ruth J. Geller, MHS ${ }^{3}$; Ashley F. Sullivan, MS, $\mathrm{MPH}^{3}$; and Carlos A. Camargo, Jr., MD, DrPH ${ }^{1,3}$.

${ }^{1}$ Division of Rheumatology, Allergy and Immunology, Department of Medicine, Massachusetts General Hospital, Harvard Medical School, Boston, MA

${ }^{2}$ Division of Pulmonary, Allergy \& Critical Care Medicine, Department of Medicine, Stanford University School of Medicine, Stanford, CA

${ }^{3}$ Department of Emergency Medicine, Massachusetts General Hospital, Harvard Medical School, Boston, MA

Address manuscript correspondence to:

Carlos A. Camargo, MD DrPH, Department of Emergency Medicine, Massachusetts General Hospital, 125 Nashua Street, Suite 920, Boston, MA 02114.

Phone: 617-726-5276 Fax: 617-724-4050

Email: ccamargo@partners.org

\section{Abbreviations:}

ASM, acid suppressant medication

CI, confidence interval

OR, odds ratio

PPI, proton pump inhibitor

H2RA, histamine-2 receptor antagonist 
GERD, gastroesophageal reflux disease

MARC-35, $35^{\text {th }}$ Multicenter Airway Research Collaboration

NHW, non-Hispanic White

\section{Key Words:}

Acid suppressant medications, allergy, prenatal exposures, bronchiolitis, food allergy, environmental allergy, asthma

Financial Disclosure: This study was supported by the grants U01 AI-087881 (Camargo), R01 AI-114552 (Camargo), and UG3 OD-023253 (Camargo) from the National Institutes of Health (Bethesda, MD). Dr Arroyo was supported by the National Institutes of Health award R25AI147369. The content of this manuscript is solely the responsibility of the authors and does not necessarily represent the official views of the National Institutes of Health.

Conflict of Interest: The authors have no conflicts of interest.

Word count: 1022 ( $\max 1200$ words)

Authors : 5 (no maximum)

Tables : 2 (3 maximum), Figures: none

References : $10(\max 10)$

\section{To the editor:}

Acid suppressant medications (ASMs), such as proton pump inhibitors (PPIs) or histamine-2 receptor antagonists (H2RAs), are commonly used during pregnancy to treat symptoms of reflux or gastroesophageal reflux disease (GERD). Prenatal exposure to ASMs is associated with increased risk of atopic disease in childhood including recurrent wheeze and asthma. ${ }^{1-3}$ The mechanism by which exposure to these medications increase risk is unknown. Increased risk of allergen sensitization has been proposed as a possible mechanism. ${ }^{4,5}$

The objective of this study was to estimate the association between prenatal exposure to ASMs and risk of allergen sensitization in early childhood.

We used data from the $35^{\text {th }}$ Multicenter Airway Research Collaboration (MARC-35), an ongoing multicenter prospective cohort study comprised of children with a history of severe bronchiolitis during infancy $(\mathrm{n}=921)$. Study design and participants have been previously described. ${ }^{6}$ Briefly, infants (age $<1$ year) were enrolled during hospitalization for bronchiolitis at 17 US hospitals during the 2011-2014 winter seasons.

Prenatal exposure to ASMs was defined as maternal use of either PPI or H2RA during pregnancy, which was obtained from parent/guardian report by questionnaire at enrollment. Parents/guardians were asked, "When pregnant with your child, did you/the biological mother take H2 blockers or proton pump inhibitors for gastroesophageal reflux (heartburn, GERD) or ulcers?" Examples were provided. Respondents were instructed not to include antacids.

Data on maternal and infant characteristics were collected by parent/guardian interview at enrollment visit during infancy. We linked infants' home address ZIP codes to median household income estimates using Esri Business Analyst Desktop (Esri, Redlands, CA). Eczema during the first year of life was determined by physician review of 2 data sources: complete medical record review for clinician-diagnosed eczema and parent report of eczema in the first year of life.

The outcome of allergen sensitization was defined by serum specific immunoglobulin E (sIgE). Serum samples from early childhood (median age 42 months, $\mathrm{n}=611$ ) were analyzed for sIgE by ImmunoCAP at the Phadia Immunology Reference Laboratory (Portage, MI). Samples were tested for food (cow's milk, egg white, peanut, cashew nut and walnut) and environmental allergens (grasses, trees, weeds, molds, cat, dog, cockroach, mouse, and dust mites). sIgE [?]0.35 kU/L was considered sensitized. We used sIgE results from 
early childhood to create several variables: food allergen sensitization (yes or no), aeroallergen sensitization (yes or no), any allergen sensitization (i.e., food or aeroallergen; yes or no). We limited analyses to those with complete exposure and outcome data $(n=594$ for analyses of any allergen sensitization and aeroallergen sensitization, $\mathrm{n}=598$ for analyses of food allergen sensitization).

We estimated the association between ASM exposure and allergen sensitization outcomes using logistic regression models. We performed unadjusted, partially adjusted (age at enrollment, sex, insurance, maternal history of asthma and season of exam) and fully adjusted models (partially-adjusted model plus median household income estimated by ZIP code, maternal history of food allergy, and geographic region of residence at time of exam). All models accounted for potential clustering by site using robust standard errors. To assess for potential effect modification, we performed stratified analysis by sex, race/ethnicity and eczema during first year of life. All analyses were performed using Stata 15.1 (Stata Corp, College Station, TX). All $\mathrm{P}$-values were two-tailed, with $\mathrm{P}<0.05$ considered statistically significant.

In this cohort, 95/598 (16\%) children were exposed to ASMs prenatally (Table 1 ). Any sensitization (food or aeroallergen) developed in 247/594 (42\%) children. The prevalence of sensitization was similar among unexposed $(207 / 499,41 \%)$ and exposed $(40 / 95,42 \%)$ (Table 2 ). Those exposed to ASM prenatally did not have increased odds of allergen sensitization (unadjusted odds ratio [OR] 1.03, 95\% CI 0.65-1.63). The association remained null after adjustment for potential confounders (fully adjusted OR 1.07, 95\% CI 0.601.91). The prevalence of food allergen sensitization was $171 / 598$ (29\%) overall, 140/503 (28\%) among the unexposed and 31/95 (33\%) among the exposed (unadjusted OR 1.26, 95\%CI 0.71-2.22). Those exposed were not at increased odds of developing food sensitization after adjustment for potential confounders (fully adjusted OR 1.38, 95\% CI 0.69-2.76). The prevalence of aeroallergen sensitization was 162/594 (27\%) overall, $137 / 499$ (27\%) among the unexposed and 25/95 (26\%) among the exposed. Those exposed to ASM did not have increased odds of aeroallergen sensitization (unadjusted OR 0.94, 95\%CI 0.57-1.56; fully adjusted OR $0.94,95 \%$ CI $0.53-1.67$ ). In analysis stratified by sex, race/ethnicity and eczema during first year of life we did not find evidence of effect modification (Supplemental Table 1 ). However, there was a trend toward increased odds among non-Hispanic Whites (NHW) for food allergen sensitization which was not observed among non-Hispanic Blacks and Hispanics.

In this study of children with history of severe bronchiolitis, we found that prenatal ASM exposure was not associated with odds of developing allergen sensitization by early childhood. Previous work in this cohort found that prenatal ASM exposure was associated with increased risk of recurrent wheezing. ${ }^{1}$ Taken together, these findings suggest that allergen sensitization may not be the sole mechanism by which prenatal ASM exposure increases risk of childhood respiratory and allergic disease.

Exposure to ASM has been associated with specific food allergen sensitization (e.g., hazelnut) in adults and animal models ${ }^{7,8}$; however, the mechanism by which prenatal ASM exposure increases risk is not known. It is clear that the human gut microbiome plays a central role in development of allergic disease. ${ }^{9}$ ASMs alter the gut microbiome, thus this remains a plausible mechanism. ${ }^{10}$

The generalizability of this study is limited as all children experienced an episode of severe bronchiolitis during infancy. While bronchiolitis is common, the patterns of allergen sensitization may differ from the general population. Future work will need to confirm these findings in healthy children.

While we did not detect significant effect modification by race/ethnicity, our data suggest a trend toward increased risk for food allergen sensitization among exposed NHW. This finding may be related to demographics of our study sample where $61 \%$ of exposed children are NHW.

In conclusion, in a cohort of children with a history of severe bronchiolitis those exposed to ASM prenatally are not at increased risk of developing food or environmental allergen sensitization by early childhood. The mechanism by which ASM exposure increases risk of childhood asthma remains unclear, but alterations in the gut microbiome merit consideration.

Sincerely, 
Lacey B. Robinson, MD, MPH

Division of Rheumatology, Allergy and Immunology, Department of Medicine, Massachusetts General Hospital, Harvard Medical School, Boston, MA

ORCID ID: 0000-0002-0008-2902

Anna Chen Arroyo, MD, MPH

Division of Pulmonary, Allergy \& Critical Care Medicine, Department of Medicine, Stanford University School of Medicine, Stanford, CA

ORCID ID: 0000-0002-7785-4632

Ruth J. Geller, MHS

Department of Emergency Medicine, Massachusetts General Hospital, Boston, MA

ORCID ID: 0000-0001-8828-0761

Ashley F. Sullivan, MS, MPH

Department of Emergency Medicine, Massachusetts General Hospital, Harvard Medical School, Boston, MA

ORCID ID: 0000-0001-6783-1720

Carlos A. Camargo, Jr., MD, DrPH

Division of Rheumatology, Allergy and Immunology, Department of Medicine, and Department of Emergency Medicine, Massachusetts General Hospital, Harvard Medical School, Boston, MA

ORCID ID: 0000-0002-5071-7654

Table 1 . Maternal and child characteristics by prenatal exposure to acid suppressant medications

\begin{tabular}{|c|c|c|}
\hline Characteristic & Total $(n=598)$ & No prenatal ASM exposure (1 \\
\hline & $\mathrm{n}(\%)$ & $\mathrm{n}(\%)$ \\
\hline Age at enrollment (months), median (IQR) & $3(2-6)$ & $3(2-6)$ \\
\hline \multicolumn{3}{|l|}{ Sex } \\
\hline Male & $352(59)$ & $296(59)$ \\
\hline Female & $246(41)$ & $207(41)$ \\
\hline \multicolumn{3}{|l|}{ Race/ethnicity } \\
\hline White, non-Hispanic & $252(42)$ & $194(39)$ \\
\hline Black, non-Hispanic & $140(23)$ & $123(25)$ \\
\hline Hispanic & $187(31)$ & $169(34)$ \\
\hline Other & $19(3)$ & $17(3)$ \\
\hline Median household income by ZIP code & Median household income by ZIP code & Median household income by ZIP \\
\hline$<\$ 40,000$ & $210(35)$ & $188(37)$ \\
\hline$\$ 40,000-\$ 79,999$ & $313(52)$ & $254(51)$ \\
\hline$\$ 80,000$ & $75(13)$ & $61(12)$ \\
\hline \multicolumn{3}{|l|}{ Insurance } \\
\hline Private & $243(41)$ & $184(37)$ \\
\hline Public or none & $353(59)$ & $317(63)$ \\
\hline Maternal history of atopic conditions & Maternal history of atopic conditions & Maternal history of atopic conditic \\
\hline \multicolumn{3}{|l|}{ Food allergy } \\
\hline No & $523(88)$ & $447(89)$ \\
\hline Yes & $72(12)$ & $53(11)$ \\
\hline Infancy & & \\
\hline
\end{tabular}




\begin{tabular}{|c|c|c|}
\hline Characteristic & Total $(n=598)$ & No prenatal AS \\
\hline Atopic dermatitis during first year of life & Atopic dermatitis during first year of life & Atopic dermatitis \\
\hline No & $466(78)$ & $393(78)$ \\
\hline Yes & $132(22)$ & $110(22)$ \\
\hline \multicolumn{3}{|l|}{ Early childhood exam } \\
\hline Age at exam (months), median (IQR) & $45(41-51)$ & $45(41-51)$ \\
\hline \multicolumn{3}{|l|}{ Geographic region* } \\
\hline Northeast & $151(25)$ & $123(25)$ \\
\hline Midwest & $59(10)$ & $42(8)$ \\
\hline South & $244(41)$ & $205(41)$ \\
\hline West & $144(24)$ & $133(26)$ \\
\hline \multicolumn{3}{|l|}{ Season of exam ${ }^{+}$} \\
\hline Spring & $172(29)$ & $147(29)$ \\
\hline Summer & $131(22)$ & $109(22)$ \\
\hline Fall & $165(28)$ & $143(28)$ \\
\hline Winter & $130(22)$ & $104(21)$ \\
\hline \multicolumn{3}{|c|}{ * Geographic regions were defined according to categories used by the U.S. Census. } \\
\hline \multicolumn{3}{|c|}{$\begin{array}{l}+ \text { Seasons were defined as: spring, Mar. } 20^{\text {th }}-\text { Jun. } 19^{\text {th }} \text {; summer, Jun. } 20^{\text {th }}-\text { Sept. } 21^{\text {st }} \text {; fall, Sept. } 22^{\text {nd }}-\text { Dec. } \\
20^{\text {th }} \text {; winter, Dec. } 21^{\text {st }}-\text { Mar. } 19^{\text {th }} .\end{array}$} \\
\hline \multicolumn{3}{|c|}{$\begin{array}{l}\text { Table 2. Association between prenatal acid suppressant medication exposure and allergic sensitization in } \\
\text { early childhood }\end{array}$} \\
\hline Outcome & \multicolumn{2}{|c|}{ Prenatal ASM exposure OR (95\%CI) } \\
\hline \multicolumn{3}{|l|}{ Any Allergen Sensitization } \\
\hline Unadjusted & $1.03(0.65-1.63)$ & \\
\hline Partially adjusted* & $1.08(0.63-1.86)$ & \\
\hline Fully adjusted $^{+}$ & $1.07(0.60-1.91)$ & \\
\hline \multicolumn{3}{|l|}{ Food Allergen Sensitization } \\
\hline Unadjusted & $1.26(0.71-2.22)$ & \\
\hline Partially adjusted* & $1.42(0.75-2.68)$ & \\
\hline Fully adjusted $^{+}$ & $1.38(0.69-2.76)$ & \\
\hline \multicolumn{3}{|l|}{ Aeroallergen Sensitization } \\
\hline Unadjusted & $0.94(0.57-1.56)$ & \\
\hline Partially adjusted* & $0.93(0.53-1.63)$ & \\
\hline Fully adjusted ${ }^{+}$ & $0.94(0.53-1.67)$ & \\
\hline
\end{tabular}

*Partially adjusted model includes age at enrollment, sex, insurance, maternal history of asthma and season of exam.

+Fully adjusted model includes age at enrollment, sex, median household income by ZIP code, insurance, maternal history of food allergy, maternal history of asthma, age at early childhood exam, geographic region of residence at exam, and season of exam.

\section{REFERENCES}

1. Robinson LB, Chen Arroyo AJ, Dantas MAS, Espinola JA, Sullivan AF, Camargo CA, Jr. Prenatal exposure to acid suppressant medications and the risk of recurrent wheeze at 3 years of age in children with a history of severe bronchiolitis. J Allergy Clin Immunol Pract. 2019 Sep-Oct;7(7):2422-2424.e4. 
2. Lai T, Wu M, Liu J, Luo M, He L, Wang X, et al. Acid-suppressive drug use during pregnancy and the risk of childhood asthma: A meta-analysis. Pediatrics. 2018 Feb;141(2):e20170889

3. Devine RE, McCleary N, Sheikh A, Nwaru BI. Acid-suppressive medications during pregnancy and risk of asthma and allergy in children: A systematic review and meta-analysis. J Allergy Clin Immunol. 2017;139(6):1985-8.e12.

4. Untersmayr E. Acid suppression therapy and allergic reactions. Allergo J Int. 2015;24(8):303-11.

5. Robinson LB, Camargo CA, Jr. Acid suppressant medications and the risk of allergic diseases. Expert Rev Clin Immunol. 2018;14(9):771-80.

6. Hasegawa K, Mansbach JM, Ajami NJ, Espinola JA, Henke DM, Petrosino JF, et al. Association of nasopharyngeal microbiota profiles with bronchiolitis severity in infants hospitalised for bronchiolitis. Eur Respir J. 2016;48(5):1329-39.

7. Untersmayr E, Bakos N, Scholl I, Kundi M, Roth-Walter F, Szalai K, et al. Anti-ulcer drugs promote IgE formation toward dietary antigens in adult patients. FASEB J. 2005;19(6):656-8

8. Untersmayr E, Schöll I, Swoboda I, Beil WJ, Förster-Waldl E, Walter F, et al. Antacid medication inhibits digestion of dietary proteins and causes food allergy: a fish allergy model in BALB/c mice. J Allergy Clin Immunol. 2003;112(3):616-23.

9. Pascal M, Perez-Gordo M, Caballero T, Escribese MM, Lopez Longo MN, Luengo O, et al. Microbiome and allergic diseases. Front Immunol. 2018;9:1584.

10. Levy EI, Hoang DM, Vandenplas Y. The effects of proton pump inhibitors on the microbiome in young children. Acta Paediatr. 2020;109(8):1531-8. 